\title{
THE INTERNAL STRUCTURE OF CORONAL MASS EJECTIONS: ARE ALL REGULAR MAGNETIC CLOUDS FLUX ROPES?
}

\author{
C. JACOBS ${ }^{1}$, I. I. Roussev ${ }^{2}$, N. LugaZ ${ }^{2}$, And S. PoedTs ${ }^{1}$ \\ ${ }^{1}$ Centrum voor Plasma-Astrofysica, Katholieke Universiteit Leuven, Celestijnenlaan 200B, 3001 Leuven, Belgium; Carla.Jacobs@wis.kuleuven.be, \\ Stefaan.Poedts@wis.kuleuven.be \\ 2 Institute for Astronomy, University of Hawai'i, 2680 Woodlawn Drive, Honolulu, HI 96822, USA; iroussev@ifa.hawaii.edu, nlugaz@ifa.hawaii.edu \\ Received 2008 November 25; accepted 2009 March 11; published 2009 April 1
}

\begin{abstract}
In this Letter, we investigate the internal structure of a coronal mass ejection (CME) and its dynamics by invoking a realistic initiation mechanism in a quadrupolar magnetic setting. The study comprises a compressible threedimensional magnetohydrodynamics simulation. We use an idealized model of the solar corona, into which we superimpose a quadrupolar magnetic source region. By applying shearing motions resembling flux emergence at the solar boundary, the initial equilibrium field is energized and it eventually erupts, yielding a fast CME. The simulated CME shows the typical characteristics of a magnetic cloud (MC) as it propagates away from the Sun and interacts with a bimodal solar wind. However, no distinct flux rope structure is present in the associated interplanetary ejection. In our model, a series of reconnection events between the eruptive magnetic field and the ambient field results in the creation of significant writhe in the CME's magnetic field, yielding the observed rotation of the magnetic field vector, characteristic of an MC. We demonstrate that the magnetic field lines of the CME may suffer discontinuous changes in their mapping on the solar surface, with footpoints subject to meandering over the course of the eruption due to magnetic reconnection. We argue that CMEs with internal magnetic structure such as that described here should also be considered while attempting to explain in situ observations of regular MCs at L1 and elsewhere in the heliosphere.
\end{abstract}

Key words: MHD - shock waves - Sun: corona - Sun: coronal mass ejections - Sun: magnetic fields

Online-only material: mpeg animation

\section{INTRODUCTION}

Coronal mass ejections (CMEs) undoubtedly belong to the most energetic transient solar events. Over the years, a plethora of CME observations have become available and the observed CME properties have been described and analyzed in great detail in many research papers (e.g., Yashiro et al. 2004; Webb et al. 2006). Most theories and models of CME initiation impart the major role in the eruption process to the magnetic field. Shearing motions along the magnetic neutral line and emergence and/or cancellation of magnetic flux, among other processes, are often observed in the source regions of CMEs around the time of the eruption. This supports the idea that the energy needed to drive an eruption must be provided through the magnetic field. Several reviews of possible CME initiation scenarios are available in the literature (e.g., Moore \& Sterling 2006; Forbes et al. 2006; Roussev 2008). All of the current CME initiation models have in common the appearance of magnetic flux ropes (FRs): they either exist before the eruption takes place, or are formed during the eruption process (Roussev \& Sokolov 2006). The main direct observational evidence linking CMEs to magnetic FRs is: (1) strong correlation between CMEs and erupting prominences, which are believed to possess some twisted magnetic field; and (2) presence of helical structures in coronagraph images of CMEs.

While traveling through interplanetary (IP) space, CMEs often develop into magnetic cloud (MC) structures that are detected in situ. Bothmer \& Schwenn (1998) have investigated 46 MCs observed with the Helios spacecraft between 0.3 and $1 \mathrm{AU}$, and they have found that in $50 \%$ of the cases the MC could be associated with a CME (see Démoulin (2008) for an up-to-date review of the link between CMEs and MCs). Since MCs are low- $\beta$ structures, a force-free field is often used to approximate their magnetic configuration. The oldest models (e.g., Burlaga 1988) approximate the MC as a cylinder and use Lundquist's solution for constant- $\alpha$ force-free magnetic fields to derive information, such as the cloud's orientation and radial extent. These models also enable the derivation of an estimate of the helicity and magnetic flux content of the MC (Lynch et al. 2003; Dasso et al. 2005). The idea of fitting the MC's in situ data with some analytical formula is already more than 20 years old and, through the years, many new models have been developed (see Riley et al. (2004) for a comparison between several flux-rope-fitting techniques). Another early model to explain observations of MCs was the magnetic plasmoid, disconnected from the Sun (see, for example, the comparison in Farrugia et al. 1995). However, Kahler \& Reames (1991) have used solar energetic particle (SEP) data to probe the magnetic topology of MCs. They have argued that the rapid access of SEPs to the interiors of many MCs indicates that the cloud field lines extend back to the Sun and, hence, that they are not plasmoids. Liu et al. (2008) have validated the FR geometry of MCs by using multiple spacecraft measurements, and they have also concluded that a MC cannot be a spherical plasmoid disconnected from the Sun. It is clear that the localized nature of the in situ spacecraft measurements are insufficient to derive the three-dimensional (3D) structure of the IP CME. Thus, numerical simulations should be regarded as complementary to observations since they can provide the missing information for the interpretation of MCs. In a recent study, Lugaz et al. (2008) have pointed out the need to combine both numerical simulations of CME events and related observations in order to achieve better interpretation of the observed phenomena.

In this Letter, we focus on investigating the internal structure of CMEs originating from complex active regions. To reach this goal, a CME is simulated within the framework of "ideal" 
3D magnetohydrodynamics (MHD). In the past, "ideal" MHD simulations have been proved successful in reproducing many of the observed features of CMEs. For example, Lugaz et al. (2007) have investigated the interaction of the multiple CME events on 2000 November 24. Also, the famous CME events on 2003 October 28 have been simulated by Manchester et al. (2008). In both cases, good agreement with relevant observations has been achieved, and the numerical results have led to better understanding of the dynamics of CMEs in IP space. These event studies have made use of data driven models for the solar wind. Input from magnetogram observations have been used to obtain a realistic reconstruction of the solar corona and solar wind, which is essential for event studies. This is because the evolution of a CME is influenced by the medium through which it propagates. These studies, however, have adopted an FR model for the CME, even though it is not clear whether FRs are always at the origin of CMEs. More complex models of CME initiation, such as that of Lynch et al. (2008), are just being integrated into a realistic background solar wind model (van der Holst et al. 2009) and have not yet been used to study the internal CME structure as it would be observed in situ by a spacecraft (e.g., Helios, ACE, etc.). This is because the inherently complex structure of the solar corona often results in a very complicated dynamics of the CME, thus making it difficult to analyze the time evolution of the simulated event. Therefore, we have opted to perform the computations in an idealized configuration of the coronal magnetic field, where the CME source region consists of a quadrupolar region nested in a global bipolar field. The eruption is triggered by applying small-scale shearing motions on the solar surface, thus resembling one consequence of flux emergence. In the next section, we describe the simulation setup in more detail. The dynamics of the modeled CME and its properties in the heliosphere are discussed in Section 3. The final conclusions of this study are summarized in Section 4.

\section{METHODOLOGY}

Roussev et al. (2007) have performed a series of numerical simulations in order to investigate the dynamics of the 2002 April 21 and August $24 \mathrm{CME}$ events. Magnetogram data from $\mathrm{SOHO} / \mathrm{MDI}$ have been used as a boundary condition for the magnetic field at the Sun, and the CME events have been initiated by stretching the opposite polarity feet of a newly emerged magnetic dipole. An important result from these simulations has been the jump-like displacements of the footprints of the erupting magnetic field in time. The present study is, in essence, a continuation of the work done by Roussev et al. (2007). Here we investigate, in more detail, the early evolution of the CME by exploring a more idealized magnetic setting for the sake of clarity. In our simulation, the bimodal structure of the solar wind is reproduced using the model of Roussev et al. (2003). The ambient solar magnetic field in the model, however, is simplified and approximated with a dipole (with a field strength of $4 \mathrm{G}$ at the poles), thus representing the physical conditions of solar minimum.

In addition to the dipole field, four subphotospheric magnetic sources are introduced, in a symmetrical fashion with respect to the solar equator, to mimic a quadrupolar-type active region. The four magnetic spots have alternating polarity, with the outer spots having a polarity opposite to that of the background dipole field (and the inner spots having the same polarity as that of the ambient dipole). The initial magnetic configuration is illustrated in Figure 1(a). The shearing motion due to the emergence of the inner spots is modeled in a manner similar to that described in Roussev et al. (2007), which follows the approach of Titov \& Démoulin (1999). The four magnetic charges are buried at a depth of $d=42,000 \mathrm{~km}$ under the solar surface. The outer charges, $q_{+}$and $q_{-}$, are separated by a distance of $2 L=135,000 \mathrm{~km}$, whereas the two inner point sources $q_{1+}$ and $q_{1-}$, are separated by a distance $L$. The center of the quadrupolar system is located at a longitude of $\varphi=90^{\circ}$, i.e., on the $Y$-axis. At the solar surface, the inner spots have a magnetic field strength of $70 \mathrm{G}$, whereas the outer spots are weaker and have a strength of $50 \mathrm{G}$. Note that quadrupolar magnetic configurations are known from observations to host a large fraction of the observed major flares on the Sun (e.g., Schmieder et al. 2006). The specific setup of the magnetic field described here yields the existence of two 3D null points (NPs) and two separatrix surfaces connected via a spine, which play an important role in the dynamic evolution of the magnetic field.

The equations of "ideal" MHD are solved using the BATS$\mathrm{R}$-US code (Powell et al. 1999). The computation is performed in a rectangular box of dimensions $([-20,20] \times[-20,20] \times$ $[-20,20]) R_{\odot}$, on a nonuniform Cartesian mesh, with grid size varying from $\Delta x=0.005 R_{\odot}$ to $\Delta x=1.25 R_{\odot}$. The grid is refined near the solar surface and in the region of the heliospheric current sheet, which coincides in this case with the solar equator, i.e., the $X Y$-plane. Additional grid refinement is applied in the active region, with $\Delta x<0.01 R_{\odot}$ up to a height of $2 R_{\odot}$. During the dynamic evolution of the CME, the grid is refined in a cone (with opening angle of $20^{\circ}$ ) around the $Y$ axis, to a level of $\Delta x=0.08 R_{\odot}$, in order to better capture the shock structure and to reduce the numerical dissipation along the path of the CME. This setup yields a total number of cells within the computational domain of the order $6.9 \times 10^{6}$. The imposed boundary conditions are the same as those used in earlier simulations of Roussev et al. (2004, 2007). At the solar surface, the density and temperature are set through the solar wind model of Roussev et al. (2003). The radial component of the magnetic field is fixed, and the flow velocity is set to zero. In the active region, the density is enhanced so that the Alfvén speed is limited to be no greater than $6200 \mathrm{~km} \mathrm{~s}^{-1}$.

\section{STRUCTURE AND EVOLUTION OF THE ERUPTING FIELD}

At time $t=0$, the inner spot in the northern (southern) hemisphere is moved toward the solar west (east) over a period of 30 minutes, at a maximum speed of $90 \mathrm{~km} \mathrm{~s}^{-1}$. This speed is at most $3 \%$ of the local value of the Alfvén speed, which results in a quasi-steady evolution of the stressed coronal magnetic field. By moving the inner magnetic sources apart, we mimic the shearing motions associated with flux emergence (Aulanier et al. 2005; Amari et al. 2007; Manchester 2007). During this phase, additional magnetic energy is pumped into the system. The rate of increase of the magnetic energy is the largest during the first 8 minutes of the shearing phase, with a maximum of $1.5 \times 10^{29} \mathrm{erg} \mathrm{s}^{-1}$; it then plateaus at $5 \times 10^{28} \mathrm{erg} \mathrm{s}^{-1}$ until the end of the shearing phase. The built-up shear increases the magnetic pressure in the system. Consequently, the closed magnetic loops in the active region start rising and the original separatrix surfaces and spine are distorted. The 3D NPs are pushed upward and aside in the direction opposite to that of the moving spots, forming two "horizontal" current sheets (HCS) at the distorted NPs. Then, the spine starts rotating counterclockwise, and magnetic reconnection at the NPs removes the overlying field. This creates the dark blue, S-shaped field line shown 


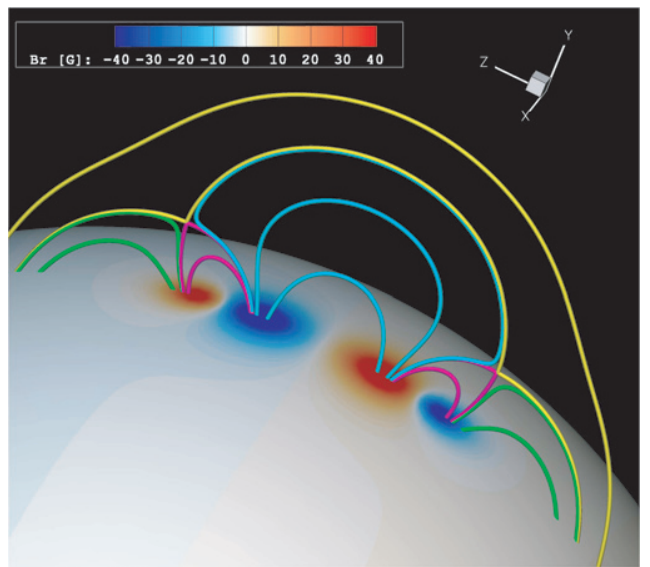

(a) $t=0 \mathrm{~min}$

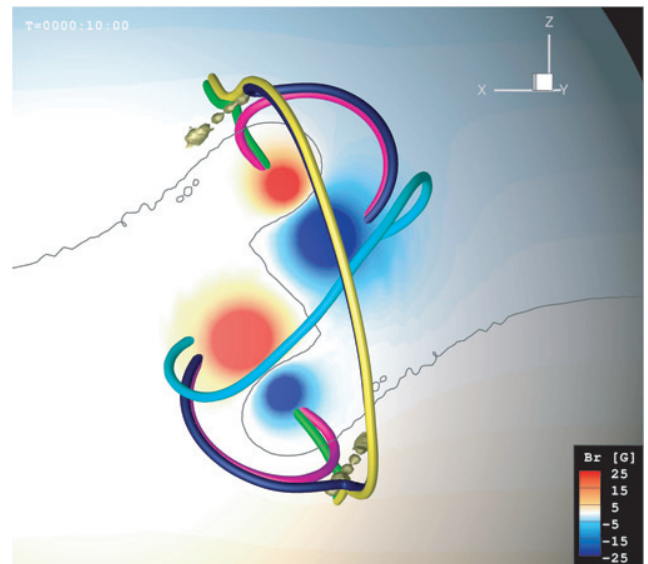

(b) $t=10 \mathrm{~min}$

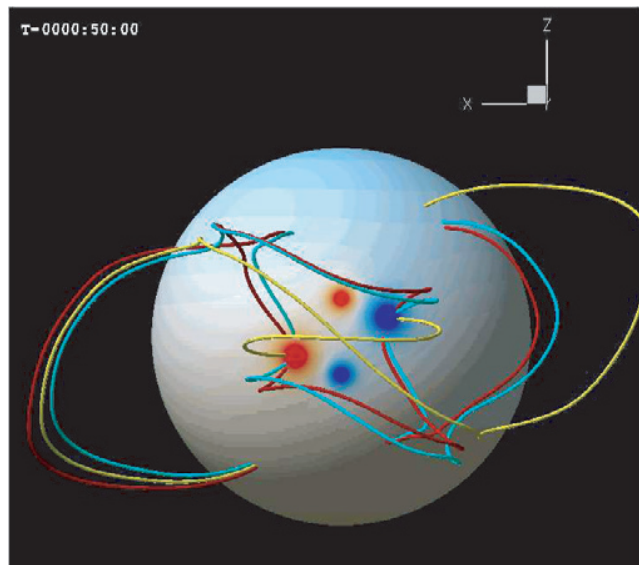

(c) $t=50 \mathrm{~min}$

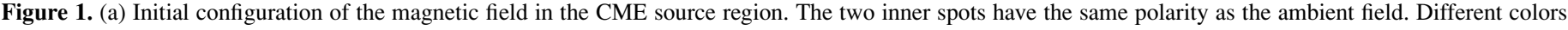

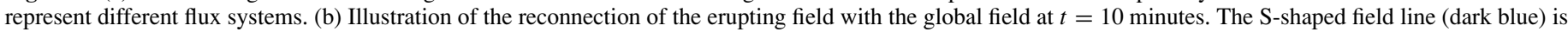

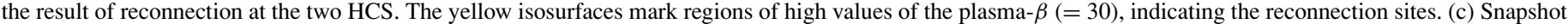

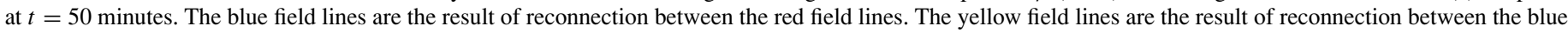

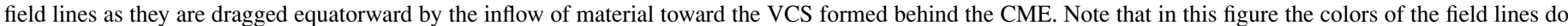
not correspond to those shown in (a). See the text for more details.

in Figure 1(b), which comprises the erupting magnetic field pushing outward. The rapid outward expansion of the erupting field (and the reduced restraining effect of the overlying field) leads to the formation of a vertical current sheet (VCS) beneath the S-shaped field lines. The sequence of reconnection events taking place at the two HCS and the VCS is illustrated in Figures 1(b) and (c).

The CME achieved in our simulation propagates with an average speed of $850 \mathrm{~km} \mathrm{~s}^{-1}$ through the corona, and the related shock wave arrives at the outer boundary of the simulation domain after $t=4.5 \mathrm{~h}$. The change in the solar wind variables, as would be measured by a satellite located at a radial distance of $15 R_{\odot}$, is shown in Figure 2 for three different vantage points in the equatorial plane. The observers are positioned along the $Y$-axis (i.e., the central direction), $15^{\circ}$, and $30^{\circ}$ away from the central direction, respectively. The IP transient passing through the satellites possesses the typical characteristics of an MC described by Burlaga \& Behannon (1982), the signal being the strongest for the central observer. We have adopted a minimum variance analysis to determine the orientation of the cloud's axis and found that it is located perpendicular to the $Y$-axis, almost coinciding with the direction of the negative $X$-axis. This is consistent with the results of Yurchyshyn (2008) who have found that the axis of MCs have the tendency to be aligned with the heliospheric current sheet. Their result might be an indication that the heliospheric magnetic field, and as such the global solar field, may have a significant influence on the solar eruption.

The standard explanation for the observed rotation of the magnetic field inside the $\mathrm{MC}$ is that the satellite is passing through a bundle of twisted field lines, or a magnetic flux rope. However, when analyzing the magnetic field structure in the simulation, we do not find a correspondence between the rotation of the magnetic field and the passage of a FR structure. Instead, what we find is that the rotation in $B_{z}$ is due to the writhe of the magnetic field rather than its twist. Figure 3 shows a top view of the CME's magnetic field structure, illustrating the rotation of the magnetic field. During the passage of the CME, the magnetic vector rotates first counterclockwise over a small angle, followed by a clockwise rotation away from the northsouth direction to reach a maximum angle of about $140^{\circ}$. The writhe of the magnetic field lines is the result of reconnection occurring in the lower corona between the erupting field and the global field, which is illustrated in Figure 1, and it involves at least three distinct reconnection sites. First, reconnection at the two HCS produces the S-shaped field lines connecting the two inner spots (see Figure 1(b)). Due to the magnetic tension force, these field lines rotate clockwise. Second, low in the corona, the 


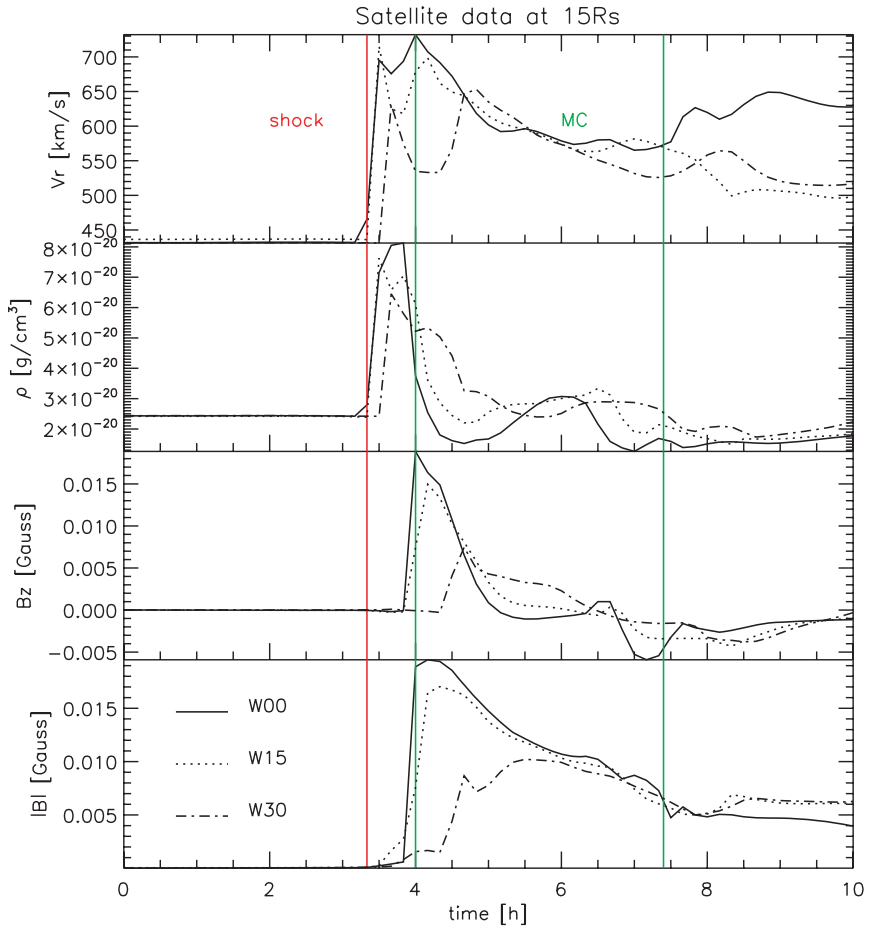

Figure 2. Plasma and IMF properties measured in situ by a satellite located at $15 R_{\odot}$ for three different observers, viz. W00, W15, and W30. The time of shock arrival and the MC are identified for the central observer (W00).

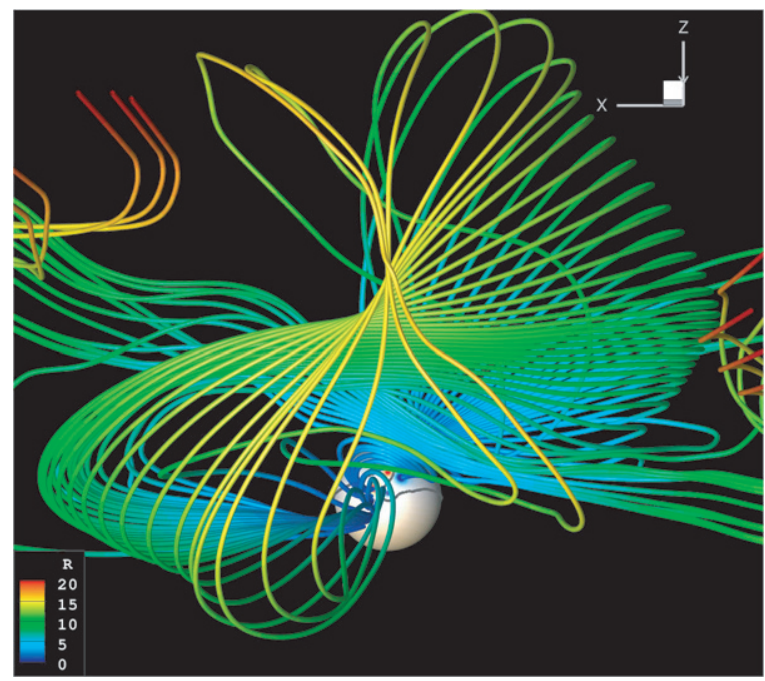

Figure 3. Top view of the erupting CME showing the 3D structure of the magnetic field at $t=4 \mathrm{~h}$. The magnetic field lines are color-coded with the radial distance from the Sun. An online movie shows the internal structure of the CME's magnetic field and the jumpwise displacement of its footprints.

(An mpeg animation of this figure is available in the online journal.)

highly stretched field connecting the two inner spots reconnects with the overlying field, thus creating the blue field lines shown in Figure 1(c). Third, as these reconnected field lines expand in height, they are dragged equatorward by the inflow of material toward the VCS formed behind the CME. As a result, they reconnect across the solar equator and form the yellow field lines shown in Figure 1(c) (note that the colors of the field lines in Figure 1(c) are not related to those in Figures 1(a) and (b)). These field lines are highly curved, and they connect the CME with very distant regions on the solar surface. The reconnection events illustrated in Figure 1(b) are different than those shown in Figure 1(c), with the latter resulting in a magnetic field vector pointing from north-east to south-west at the location of the satellite. The result of all of these reconnection events is: (1) creation of two systems of flare loops connecting the active region with the quiet Sun; and (2) magnetic connection between very remote regions on the Sun. A very important finding in the present simulation is that the magnetic field lines of the CME suffer discontinuous changes in their mapping on the solar surface, with footpoints subject to meandering over the course of the eruption due to magnetic reconnection. This is illustrated in the mpeg animation accompanying Figure 3.

\section{CONCLUSIONS}

In this Letter, we have presented an alternative picture for the internal structure of a fast CME, which is different from the classical FR scenario. We have found that the presence of two 3D NPs in the initial configuration is essential for yielding a fast CME that has a significant amount of writhe in the magnetic field. Although the simulated eruption shows the typical features of an MC, we have demonstrated that the rotation of the magnetic field vector inside the CME is not due to the passage of a magnetic FR. In our simulation, the rotation in the vertical component of the magnetic field, $B_{z}$, is caused by a series of reconnection events between the erupting field and the global field of the quiet Sun. This results in jumps of the magnetic footprints of the CME to very distant regions on both hemispheres of the Sun. Assuming that there are no inhomogeneities in the solar wind, the CME will undergo a self-similar expansion that would yield a qualitatively similar internal structure of the MC at greater heliospheric distances. This, however, remains to be proved in a follow-up investigation in which the CME will be evolved out to $1 \mathrm{AU}$ and beyond.

Note that reconnection at multiple-sites and four-ribbon flares is often present in the most violent solar eruptions, for example the X17 solar flare of 2003 October 28 (Schmieder et al. 2006). This event was preceded by the emergence of a new dipole in an existing active region. Large-scale, multisite reconnection removed part of the overlying field, yielding a major eruption, which was associated with a classical two ribbon flare. As another example, the eruptive event on 2004 January 20 was also accompanied by four-ribbon flares (van Driel-Gesztelyi et al. 2008). This observation is consistent with our simulation results too. Both eruptive events were also associated with coronal dimmings. Attrill et al. (2007) have interpreted the coronal dimmings as large-scale reconnections between the expanding field of the CME and the ambient magnetic field. As a consequence, the footprints of the CME can spread over a wide portion of the solar surface, as confirmed in an observational study of Zhang et al. (2007), who have concluded that the largescale magnetic field of the Sun has to be regarded as the real source of CMEs. The large shifts in the location of the magnetic footprints of the CME, as seen in our simulation, have also been established in the event studies of Roussev et al. (2007) and in the simulation of Gibson \& Fan (2008). Both studies have shown that the feet of the erupting FR have lost connection with the CME source region. This may have implications for the production of SEPs, and it should result in changes in the observed plasma composition when a MC passes through a spacecraft.

Our simulation has also demonstrated that whether or not a FR is present in the CME strongly depends on the boundary motions applied to trigger the eruption. In general, simulations using rotating motions to energize the magnetic field do report 
on the formation of magnetic FRs (DeVore \& Antiochos 2008; Lynch et al. 2008; Titov et al. 2008). In a future study, the CME initiation mechanism will be applied to different configurations of the initial magnetic field, and we will investigate whether or not the same conclusions still hold.

C.J. would like to acknowledge the warm hospitality she received during her six month visit at the IfA. The authors would like to thank Klaus Galsgaard, Igor Sokolov, and an anonymous referee for the useful comments made on the manuscript. The research at the CPA has been supported by the projects GOA/2009-009 (K. U. Leuven), G.0304.07 (FWO Vlaanderen), C 90205 (ESA Prodex 9), and by the SOLAIRE Network (MTRN-CT-2006-035484). The research at the IfA has been supported by the grants: NSF ATM-0454469 (FDSS), NSF ATM-0631790 (SHINE), NSF ATM-0639335 (CAREER), NASA NNX07AC13G (LWS), and NNX08AQ16G (LWS).

\section{REFERENCES}

Amari, T. , Aly, J. J., Mikić, Z., \& Linker, J. 2007, ApJ, 671, L189

Attrill, G. D. R., Harra, L. K., van Driel-Gesztelyi, L., \& Démoulin, P. 2007, ApJ, 656, L101

Aulanier, G., Pariat, E., \& Démoulin, P. 2005, A\&A, 444, 961

Bothmer, V, \& Schwenn, R. 1998, Ann. Geophys., 16, 1

Burlaga, L. F. 1988, J. Geophys. Res., 93, 7217

Burlaga, L. F., \& Behannon, K. W. 1982, Sol. Phys., 81, 181

Dasso, S., Mandrini, C. H., Démoulin, P., Luoni, M. L., \& Gulisano, A. M. 2005, Adv. Space Res., 35, 711

Démoulin, P. 2008, Ann. Geophys., 26, 3113

DeVore, C. R., \& Antiochos, S. K. 2008, ApJ, 680, 740

Farrugia, C., Osherovich, V., \& Burlaga, L. 1995, J. Geophys. Res., 100, 12293
Forbes, T. G., et al. 2006, Space Sci. Rev., 123, 251

Gibson, S. E., \& Fan, Y. 2008, J. Geophys. Res., 113, A09103

Kahler, S. W., \& Reames, D. V. 1991, J. Geophys. Res., 96, 9419

Liu, Y., et al. 2008, ApJ, 677, L133

Lugaz, N., Manchester, W. B., Roussev, I. I., Tóth, G., \& Gombosi, T. I. 2007, ApJ, 659, 788

Lugaz, N., Vourlidas, A., Roussev, I. I., Jacobs, C., Manchester, W. B., \& Cohen, O. 2008, ApJ, 684, L111

Lynch, B., Antiochos, S. K., DeVore, C. R., Luhmann, J. G., \& Zurbuchen, T. H. 2008, ApJ, 683, 1192

Lynch, B., Zurbuchen, T. H., \& Fisk, L. A. 2003, J. Geophys. Res., 108, 1239

Manchester, W. B. 2007, ApJ, 666, 532

Manchester, W. B., et al. 2008, ApJ, 684, 1448

Moore, R. L., \& Sterling, A. C. 2006, in AGU Monograph Series 165, Solar Eruptions and Energetic Particles, ed. N. Gopalswamy, R. Mewaldt, \& J. Torsti, 43

Powell, K. G., Roe, P. L., Linde, T. J., Gombosi, T. I., \& DeZeeuw, D. L. 1999, J. Comput. Phys., 154, 284

Riley, P., et al. 2004, J. Atmos. Sol. Terr. Phys., 66, 1321

Roussev, I. I. 2008, J. Contemp. Phys., 49, 237

Roussev, I. I., Lugaz, N., \& Sokolov, I. V. 2007, ApJ, 668, L87

Roussev, I. I., \& Sokolov, I. V. 2006, in AGU Monograph Series 165, Solar Eruptions and Energetic Particles, ed. N. Gopalswamy, R. Mewaldt, \& J. Torsti, 89

Roussev, I. I., et al. 2003, ApJ, 595, L57

Roussev, I. I., et al. 2004, ApJ, 605, L73

Schmieder, B., et al. 2006, Adv. Space Res., 37, 1313

Titov, V. S., \& Démoulin, P. 1999, A\&A, 351, 707

Titov, S. V., Mikić, Z., Linker, J. A., \& Lionello, R. 2008, ApJ, 675, 1614

van der Holst, B., et al. 2009, ApJ, 693, 1178

van Driel-Gesztelyi, L., et al. 2008, Adv. Space Res., 42, 858

Webb, D., et al. 2006, J. Geophys. Res., 111, A12101

Yashiro, S., et al. 2004, J. Geophys. Res., 109, A07105

Yurchyshyn, V. 2008, ApJ, 675, L49

Zhang, Y., et al. 2007, Sol. Phys., 241, 329 